\title{
TREMA: A traffic-aware energy efficient MAC protocol to adapt the LoRaWAN capacity
}

\author{
Laurent Chasserat, Nicola Accettura, Balakrishna Prabhu and Pascal Berthou \\ LAAS-CNRS, Université de Toulouse, CNRS, UPS, Toulouse, France \\ Email: \{firstname.lastname\}@laas.fr
}

\begin{abstract}
The emerging LoRa technology is quickly becoming the de facto standard for Low Power Wide Area Networks upon unlicensed frequencies. Herein, the LoRaWAN medium access sets up a lightweight network architecture able to connect very low power devices to the Internet. Traffic flows in such deployments can be variable, or even unpredictable, depending on the needs of the monitoring applications using the network. As an example, to track air quality in cities, some applications can trigger an increased need of fine grained pollution data during the daytime. However, the network capacity is currently limited by the default LoRaWAN pure ALOHA access scheme. A time synchronized scheduled access would considerably improve the achievable throughput, at the cost of an increased power consumption for synchronization duties. In such a context, this contribution introduces the traffic-aware energy efficient Medium Access Control (TREMA) protocol for LoRa networks, capable of seamlessly switching between asynchronous and synchronous schemes according to the probed traffic variations. TREMA ultimately increases the maximum capacity of LoRa deployments while always selecting the most energy efficient access scheme.
\end{abstract}

\section{INTRODUCTION}

In the last decade we have witnessed relevant technological progresses in radio communications and electronic miniaturization techniques. Together they unveiled the possibility to monitor physical phenomena at large scale [1]. Low power wireless devices have been then leveraged to deploy widely spread radio networks. However, battery replacement can be very expensive, and in most cases unfeasible in networks with potentially thousands of devices, so maximizing their lifetime becomes the only viable solution. Herein, the Low Power Wide Area Network (LPWAN) architecture has been largely adopted for long range, low throughput and energy efficient data collection with relatively simple and cheap devices. Several LPWAN technologies have emerged, including Long Range (LoRa) networks. They are promoted by the LoRa Alliance, which is a consortium gathering more than 500 companies to drive the open development of the LoRa Wide Area Network (LoRaWAN) specification [2]. LoRa deployments display many interesting features such as bidirectional communications and end-to-end encryption, making them suitable for a large variety of applications. Moreover, LoRaWANs are built upon unlicensed Industrial, Scientific, Medical (ISM) radio bands enabling easy prototyping and deployment.

The LoRaWAN Medium Access Control (MAC) scheme was designed as pure ALOHA for uplink communications, meaning that upon frame generation, devices immediately start transmitting without checking whether the radio channel is free. Such a random access scheme does not require synchronization, thus saving power. However, this scheme suffers from high collision rates that naturally limit the channel throughput to $18 \%$ at most [3]. A low throughput is thus traded-off for a low power consumption. Several works in the literature have proposed channel access improvements to increase the scaling capabilities of LoRa, many of them based on synchronization and the consequent possibility to slice time into slots. Such enhancement allows to increase the maximum throughput, but we showed in a previous contribution [4] that the additional energy consumption induced by the process only makes it beneficial for high traffic situations.

In short, pure ALOHA is more energy efficient when the traffic load is low, and the synchronized access is preferable for high frame generation rates. Therefore, in scenarios where the traffic load varies over time, the access scheme should be adapted dynamically in order to maximize the lifetime of batteries. As an example, one of the many LoRaWAN applications is pollution tracking. Interestingly, the air quality in a city varies much more during the daytime than during the night [5]. Efficient monitoring of this kind of phenomena would thus require a time-varying number of measures. In the considered example scenario, switching between the asynchronous pure ALOHA access scheme and a time-synchronized one would permit a higher data reporting rate during daytime, and conversely energy savings during night hours.

In such a context, this paper timely presents the trafficaware energy efficient MAC protocol (TREMA), able to seamlessly switch between access modes depending on the probed conditions. In order to fully exploit the capabilities of TREMA in high traffic scenarios, a time-synchronized scheduled access relying on the LoRaWAN beaconing system is introduced as well. In addition, this contribution also features a beacon skipping strategy able to save energy while ensuring that cumulative clock drifts do not generate transmission misalignment. Remarkably, configuring TREMA requires the deployment fingerprint, defined as the characterization of the metrics detailed in Section III-B as a function of the traffic load for the considered network. This prior performance evaluation is carried-out for both the asynchronous and synchronous access schemes, and is then leveraged by TREMA to maximize energy efficiency in all situations.

In more details, the design of TREMA includes $(i)$ a timesynchronized scheduled access to be used when the traffic load is high, (ii) a detailed description of the required deployment 


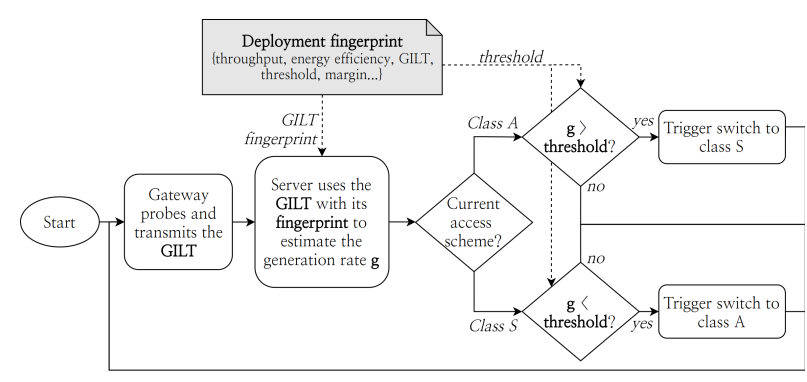

Fig. 1: TREMA's switching mechanism flowchart

fingerprint, (iii) a probing strategy that aims at estimating the generated traffic, (iv) a decision mechanism that determines whether or not a switching should be triggered, and $(v)$ the signaling protocol required to adapt the access scheme of all devices from asynchronous to synchronous and vice versa.

The performance of such a protocol is assessed through simulations. To this aim, a preliminary analysis is done on a large scale single-gateway LoRa deployment to figure out its fingerprint in terms of expected throughput and energy efficiency. Then, TREMA is tested in an example scenario to show how the network capacity is adapted in reaction to traffic load variations. A final performance assessment has been pursued to feature its behavior under any traffic condition. To the best of the authors' knowledge, TREMA is the first protocol aiming at dynamically synchronizing and desynchronizing LoRa deployments according to the traffic load variations, thus adapting the network capacity and always maximizing energy efficiency.

The rest of this work is organized as follows. Section II highlights other significant contributions about medium access enhancements for LoRa networks. Section III presents the design of TREMA in detail. In Section IV, a simulation environment is used to assess the performance of TREMA. Finally, Section V concludes this contribution and envisages research perspectives and future works.

\section{RELATED WORK}

The design of efficient MAC layer enhancements to the pure ALOHA LoRaWAN uplink scheme is a hot research topic, as clearly pictured in what follows. If some Carrier Sense strategies have been explored [6], most of the novel protocols somehow rely on synchronization and the induced possibility to slice time into transmission slots. A first work in this direction has been provided by Rizzi et al. in [7], where class A transactions are placed within a coarse Time Slotted Channel Hopping scheme to reach real-time requirements. Yet, the slot size is not tailored to maximize the throughput and no explanation is provided on how to achieve synchronization.

There are several ways to give all devices a common time reference, and they can be used to discriminate some of the proposed protocols. For instance in [8] and [9], respectively proposing a slotted ALOHA and a scheduling scheme over LoRaWAN, acknowledgements are used to synchronize the devices. This option has not been chosen here because the gateway DC limitations and collisions induce a poor downlink reliability [10], which may lead to synchronization difficulties when increasing the network load. The authors of [11] proposed to use low-power wake-up receivers to setup an on-demand Time Division Multiple Access (TDMA) that completely avoids frame collisions. In [12], FM-RDS is used to synchronize the devices out-of-band and enable a slotted ALOHA access. The main drawback in these two contributions is that they require adding specific circuitry to the devices. In [13], a time-slotted scheme is achieved by using a Synchronization and ACKnowledgement (SACK) packet, requiring dramatic changes to the legacy protocol. Interestingly, a recent contribution [14] proposed a method to temporarily alter the protocol in order to perform firmware updates. This is a first example showing that the MAC layer can be changed to suit the context. In a previous contribution, we proposed to rely on the LoRaWAN beacons for synchronization. In that, we defined Class $S$ as an extension of Class $B$ in order to introduce uplink transmission timeslots fitting the longest frame time-onair of the network [4]. The beacons were also leveraged in [15], with the goal of providing a contention-free access during a sub-portion of the beacon period. Yet, the goal there was to reserve channel resources to critical traffic, not to increase the overall network capacity. Compared to the alternatives, Class $S$ has the advantages of requiring very little changes to the legacy protocol and keeping the device complexity low. It is therefore the basis on which we build the scheduling scheme used throughout this contribution.

\section{DESIGN OF TREMA}

The goal of TREMA is to adapt the LoRaWAN capacity to the traffic load while always maximizing energy efficiency. Herein, a time-synchronized scheduled access is presented to increase the achievable throughput. It provides a good energy efficiency when the generated traffic is high. On the other hand, the legacy pure ALOHA asynchronous scheme is more efficient when the generated traffic is low. TREMA thus implements a mechanism capable of seamlessly switching between the two aforementioned schemes depending on the situation. To achieve this goal, the radio medium is probed to estimate the current traffic load. The network server then uses this estimation to select the most appropriate access scheme. To do so, this decision mechanism refers to a pre-established deployment fingerprint that relates the network behavior under different traffic loads. When the access scheme needs to be changed, the signaling protocol embedded in TREMA is leveraged to transmit switching commands to the end devices. This behavior is pictured in the flowchart of Figure 1.

\section{A. A time-synchronized scheduled access over Class $S$}

The proposed time-synchronized resource scheduling protocol is built upon Class $S$, a TDMA scheme already introduced in a previous contribution [4]. Class $S$ takes advantage of the Class $B$ synchronization beacons broadcast by the gateways to define transmission timeslots. These slots fit the longest frame time-on-air of the considered deployment to ensure that 
collisions may only occur between frames transmitted within the same slot. The highest priority in LoRa networks is to maximize the lifetime of the device batteries. The transmission time must therefore be minimized in order to reduce the energy required to send a frame and the collision probability. This is achieved by using the highest LoRaWAN data rate available, DR5, which corresponds to the smaller SF. Results can however safely be extended to the other orthogonal SFs by following a similar reasoning to further increase the capacity. With DR5, the maximum LoRaWAN MAC payload allowed (250 bytes, with the Coding Rate set to 4 and explicit header and Message Integrity Code enabled) has a time-on-air of $626.94 \mathrm{~ms}$ for data frames and $173.06 \mathrm{~ms}$ for beacons [16]. In order to comfortably fit this frame length, the LoRaWAN BEACON_WINDOW is divided into 187660 ms-long Class S timeslots. In fact, this length is a multiple of the $30 \mathrm{~ms}$ LoRaWAN receive window, so that hardware implementations will require minimal modifications to the firmware.

Given the focus on delay tolerant applications that require only unconfirmed uplink traffic in a single-gateway deployment, a simple resource scheduling scheme has been designed to maximize the achievable network throughput. Specifically, all devices are assigned to a specific slot within the slot frame based on their network joining index. This guarantees that all devices are evenly spread out between all available slots. Each device is thus competing with less neighbors, but for a shorter duration. The frame collision probability is therefore lower than when using slotted ALOHA if all devices are active and generating frames at similar rates. A round-robin channel hopping is used to prevent a possible channel fading from making a given device unreachable.

\section{B. Prior fingerprinting of the deployment}

In order to wisely select the most energy efficient access scheme for a given network load, it is beforehand necessary to study the deployment behavior for each scheme depending on the frame generation rate $g$. This analysis ultimately allows to derive $g$ from the probed traffic information, and provides a threshold value for $g$ which allows to decide which access scheme should be chosen. The use of this deployment fingerprint in TREMA's switching mechanism is depicted in the flowchart of Figure 1. The throughput $T$, expressed in bytes per second, represents the amount of data successfully transmitted during a given time interval. It must be assessed in order to understand the maximum capacity of the network. The Gateway Idle Listening Time (GILT) metric is defined as the average time when the gateway is not receiving any packet, computed over all uplink channels. It plays a role in the traffic load estimation, which is detailed hereafter in Section III-C. The energy efficiency $E$ [17] is obtained by dividing the throughput $T$ by the average power consumption. In order to increase $E$, devices may skip some of the synchronization beacons. Indeed, the LoRaWAN specification states that devices should be able to maintain beacon-less operation during at least two hours if the RX slots are widened according to the worst-case drift time since the last synchronization. Hereafter, this upper bound to the allowed drift is indicated with drift $_{\max }$, while the parameter $n_{\text {skip }}$ is introduced to represent the number of beacons skipped by devices. For instance, if $n_{\text {skip }}=1$, the device will listen to one beacon out of two. The relationship between the maximum time-on-air within the network and the chosen Class $S$ slot size determines drift $_{\text {max }}$, which in turn bounds the maximum value for $n_{\text {skip }}$. As we will show later on in Section IV-A, the fingerprint of $E$ allows to determine a traffic load tipping point, under which pure ALOHA is the most energy efficient access scheme, and above which the synchronized access becomes preferable. This tipping point defines the frame generation rate threshold guiding TREMA's decision mechanism.

\section{Network probing and decision mechanism}

TREMA's probing strategy aims at estimating the generated traffic thanks to a measurable value. The probed metric must respect two conditions: (i) the generated traffic should be deductible from its value, and (ii) all gateways must be able to compute it easily. The GILT is an excellent choice for this task, as it checks both requirements. Condition $(i)$ is respected by any metric that proves to be a bijective function of the considered generation rate range. Indeed, the GILT metric strictly decreases when the traffic load increases. The GILT also matches (ii), as all gateways can keep track of their idle time. This is why this metric was chosen over the collision time for instance, that checks $(i)$ as well, but would require discriminating the successes from the failures among the total reception duration. It is interesting to note that the introduction of the GILT within the fingerprint was necessary, as the $T$ matches only (i), while $E$ complies only with (ii). The gateway keeps track of the GILT and transmits it to the server periodically. Upon reception of the probed GILT, the server uses it to deduce an estimation of the offered traffic with the GILT fingerprint. Such an estimate is checked against the threshold value to decide whether the access scheme should be adapted following the reasoning depicted in Figure 1.

\section{Signaling protocol}

It will be shown in Section IV-A that the transient states in which access schemes coexist are sub-optimal in terms of energy efficiency. This contribution will therefore focus on switching between $100 \%$ Class $A$ and $100 \%$ Class $S$ scenarios. The switching command requires the definition of a SwitchCommand MAC command that will be transmitted through the FOpts field within the MAC payload [2]. As there are only two possible access modes for now, one bit will suffice to transmit the command value. When switching from Class $A$ to Class $S$, the Class A RX windows are used to transmit the switching commands. The server must therefore wait for an uplink transmission from a given device before triggering its class switching. This signaling induces a lot of downlink traffic and may take some time to converge depending on the device transmission rate, but it is expected to be more energy efficient than forcing devices to track beacons to determine what access scheme they should be using. This 


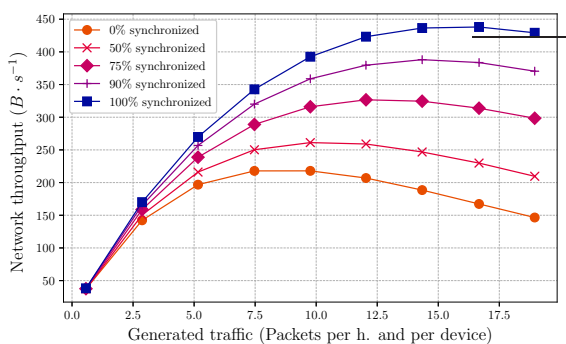

(a) Throughput.

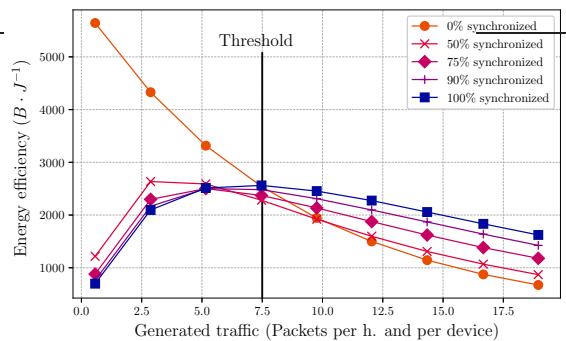

(b) Energy efficiency $\left(n_{\text {skip }}=0\right)$

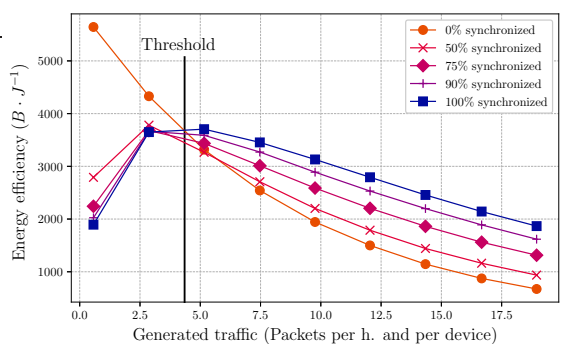

(c) Energy efficiency $\left(n_{\text {skip }}=3\right)$.

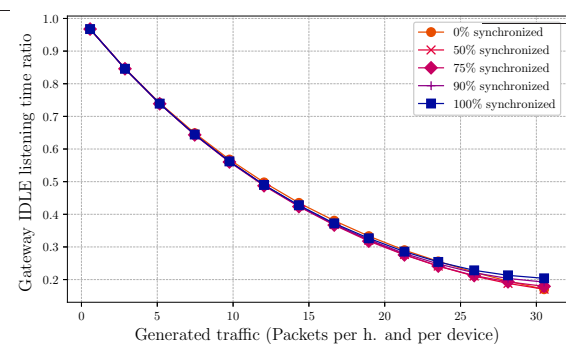

(d) GILT.

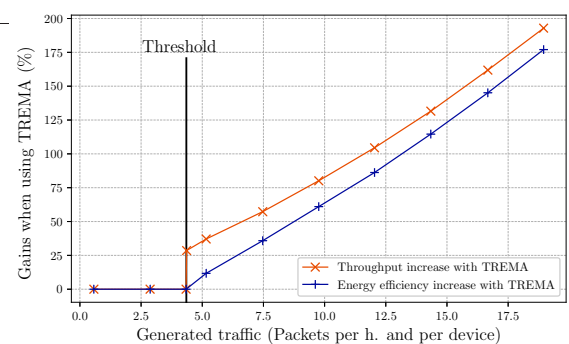

(e) Increase in $T$ and $E\left(n_{\text {skip }}=3\right)$.

Fig. 2: Fingerprint of the simulated deployment as a function of the generated traffic

aspect will be proved in future contributions. The switching process can however be sped up in the particular case where devices are switched from Class $S$ to Class $A$. Indeed, all synchronized devices receive beacons periodically, so using them to broadcast a desynchronization command allows the process to converge much faster.

\section{Performance eVAluation}

In order to assess the goodness of the TREMA design, an ad-hoc event-based simulator has been used. To kick off TREMA, the deployment fingerprint was established beforehand through large simulation campaigns using the considered network parameters. This allowed to quantify the achieved performance gain. After that, an example scenario in which the frame generation rate varies over time has been used to feature the online TREMA behavior.

The network features have been set to resemble a large scale single-gateway deployment of 1000 devices. All frames are considered to be featured with the maximum time-on-air allowed for DR5, $626.94 \mathrm{~ms}$ (cf. Section III-A). All devices use homogeneous rates in each scenario, which is a reasonable simplification as the fingerprint is established as a function of the overall traffic. Herein, the simulator frame-arrival process is modeled as a Poisson process with a variable rate to represent the fluctuations of the aggregated load. These rates vary from $\sim 0.5$ to $\sim 20$ packets per hour. The traffic is restrained to uplink data communications only, thus the downlink data frames have been disabled along with acknowledgements and re-transmissions. In this way, the downlink capabilities are left free to be used without interference by the switching mechanism. Impact of downlink traffic on switching delays and overall performances will be tackled in future works. Only the three mandatory LoRaWAN channels are implemented, which are subject to a $1 \%$ Duty Cycle.

\section{A. Preliminary deployment fingerprinting}

In order to assess which access scheme performs better for different traffic loads, the deployment fingerprint was established when using Class $A$ and the scheduled access built over Class S. Different degrees of coexistence of the two modes were also assessed to evaluate the network behavior during the transient states of the switching For the sake of statistical significance, for each configured scenario, 10 different realizations have been considered by feeding the pseudorandom number generator with 10 different seeds. $T$ has been plotted in Figure 2a, for different percentages of synchronized devices. The figure shows that for this deployment, Class $A$ is capable of offering a maximum of $220 B \cdot s^{-1}$, while the scheduled access peaks at $440 B \cdot s^{-1}$. It therefore provides a $100 \%$ capacity increase, which is better than the $88 \%$ increase offered by slotted ALOHA over Class $S$ in [4].

Another notable observation is that the advantage of the scheduled access is evident when a significant portion of the devices are synchronized. Indeed, a capacity increase of solely $18 \%$ is observed when $50 \%$ of devices operate over Class $S$, and with $90 \%$ of synchronized devices the curve peaks at $390 \mathrm{~B} \cdot \mathrm{s}^{-1}$ which corresponds to a relative improvement of simply $77 \%$. When using Class $S$, the chosen slot size sets the value of $d$ rift $_{\max }$, and therefore an upper bound for $n_{\text {skip }}$, because any transmission overlapping onto another slot should be strictly prohibited. A typical low-cost LoRa device crystal has a 30 ppm quality, which means that in the worst-case scenario, it may drift of $1 \mathrm{~ms}$ every $33.3 \mathrm{~s}$. With our parameters, the transmission is framed by a margin of rrift $_{\max }=$ $(660-626.94) / 2=16.53 \mathrm{~ms}$ within its slot. It would take 
a minimum of 550.5 seconds to get a similar drift, so the maximum number of synchronization-less beacon periods that a device can maintain is $\lfloor 550.5 /$ BEACON_PERIOD $\rfloor=4$, which bounds $n_{\text {skip }}$ to 3 . A higher energy efficiency may be achieved by widening the slots to enlarge the margin, but this would have the effect to reduce the maximum achievable throughput. Once again, designing the deployment requires trading-off network capacity for battery life. From these considerations, $E$ has been plotted for the minimum and maximum values of $n_{\text {skip }}$, respectively 0 and 3 , in Figure $2 \mathrm{~b}$ and $2 \mathrm{c}$. When using the maximum value, the RX slots opened for beacon receptions have been widened by $w$, defined as:

$$
w=2 \cdot n_{\text {skip }} \cdot \mathrm{BEACON} \_\mathrm{PERIOD} \cdot 3 \cdot 10^{-5}
$$

With $3 \cdot 10^{-5}$ representing the $30 \mathrm{ppm}$ crystal quality, $n_{\text {skip }} \cdot$ BEACON_PERIOD the synchronization-less time induced by the skipping, and the multiplication by 2 accounts for a possible positive or negative drift. TREMA aims at maximizing the energy efficiency at all times. A first remark that could be made on Figure 2 is that the maximum value for $E$ is always attained with either $0 \%$ or $100 \%$ of synchronized devices. Therefore, the coexistence of the two access schemes should remain transitory. The point at which the $0 \%$ and $100 \%$ curves cross defines the frame generation rate threshold over which the scheduled access becomes more energy efficient than pure ALOHA. This is the tipping point used by TREMA to decide whether the network should be synchronized or not. An interesting impact of setting $n_{\text {skip }}$ to 3 is that it shifts the threshold to the left. Indeed, without beacon skipping, the scheduled scheme becomes more efficient than pure ALOHA when generating $7.5 \mathrm{pkt} / \mathrm{h}$ per device, but when receiving only 1 beacon out of 4 the threshold is reduced to $4.5 \mathrm{pkt} / \mathrm{h}$ per device. This allows to profit from the gain in network capacity induced by synchronization at a lower rate, and therefore to ameliorate the frame delivery ratio between 4.5 and $7.5 \mathrm{pkt} / \mathrm{h}$ per device. Finally, the GILT fingerprint has been plotted in Figure 2d. With the specific parameters used in this deployment, curves for all class divisions overlap. However in the general case, the network class division is used to interpolate the appropriate traffic load from the closest class division curves. Further studies about the impact the network parameters on the GILT shall be led in future works.

In order to quantify the performance gain achieved when using TREMA compared to the legacy LoRaWAN access, the increase in terms of throughput and energy efficiency has been plotted in Figure 2e as a function of the generated traffic, when using $n_{\text {skip }}=3$. When the rate is below the $4.5 \mathrm{pkt} / \mathrm{h}$ per device threshold, the gains are $0 \%$ because TREMA selects the Class A. However, when the rate is above the threshold, the relative gains increase because the synchronized access performs better than pure ALOHA with these high traffic loads. The energy efficiency curve is continuous because the $100 \%$ and $0 \%$ synchronized curves cross in the $E$ fingerprint, which is not the case for the throughput.

\section{B. Online testing}

In order to test TREMA, a 24 hour scenario has been simulated to employ day and night frame generation rates. In the interval between midnight and $8 \mathrm{AM}$, a low rate of 1.5 $\mathrm{pkt} / \mathrm{h}$ per device is used, lower than the switching threshold. Then, the devices employ a higher rate of $10 \mathrm{pkt} / \mathrm{h}$ per device until $8 \mathrm{PM}$, higher than the tipping point, and finally go back to the night rate. No beacons are skipped, therefore the threshold is $7.5 \mathrm{pkt} / \mathrm{h}$ per device. The network behavior is evaluated through the plotting of $T$ and $E$ over time using a sliding window averaging over the last 15 minutes, with and without the switching mechanism. When enabled, the number of devices using each access mode is plotted over time as well. All these test results are pictured in Figure 3.

Without TREMA, the network always operates with Class $A$. When the rate increases, the throughput stabilizes at $220 B \cdot s^{-1}$ and the energy efficiency at $2000 B \cdot J^{-1}$, which were indeed the values observed for the $0 \%$ synchronized fingerprint curves in figs. $2 \mathrm{a}$ and 2 for $10 \mathrm{pkt} / \mathrm{h}$ per device. But, when switching is enabled, the rate increase is automatically detected by the server that starts synchronizing the devices. The process is not instantaneous, and takes about 3 hours, as seen in Figure 3a. This transition time explains the tipping point observed at around $9 \mathrm{AM}$ in the energy efficiency curve. Once again these values are perfectly consistent with the fingerprint data. This time, the throughput $T$ remains stable at $400 B \cdot s^{-1}$, while the energy efficiency $E$ sets up at $2500 B \cdot J^{-1}$. When the rate decreases at nightfall, the desynchronization beacon allows to switch the whole deployment back to Class $A$ instantly, and the metrics return to their initial values. Two additional remarks can be made about the $E$ plot. First, the energy efficiency drops faster when the rate increases if TREMA is enabled. This is due to the additional energy consumption induced by the reception of the switching commands. On the other hand, when the rate decreases, $E$ increases a bit later when TREMA is enabled than when it is disabled. This is because the system takes some time to probe the traffic load reduction and trigger the switching to Class $A$.

\section{CONCLUSIONS AND FUTURE WORKS}

The TREMA protocol was developed to dynamically adapt the LoRaWAN MAC layer to traffic load variations. More specifically, the legacy pure ALOHA is used when the generated traffic is low, and the access is switched to a time-synchronized scheduling of the transmissions when the network gets more congested. A threshold-based decision mechanism is used to seamlessly switch between the schemes according to the probed traffic load. Results show that this mechanism increases the maximum achievable throughput while always maximizing the device energy efficiency, by synchronizing and desynchronizing the devices. One limitation of this preliminary approach is that it requires large simulation campaigns to establish the deployment fingerprint before implementing the mechanism. Future contributions will evaluate the tools capable of establishing such fingerprints on 


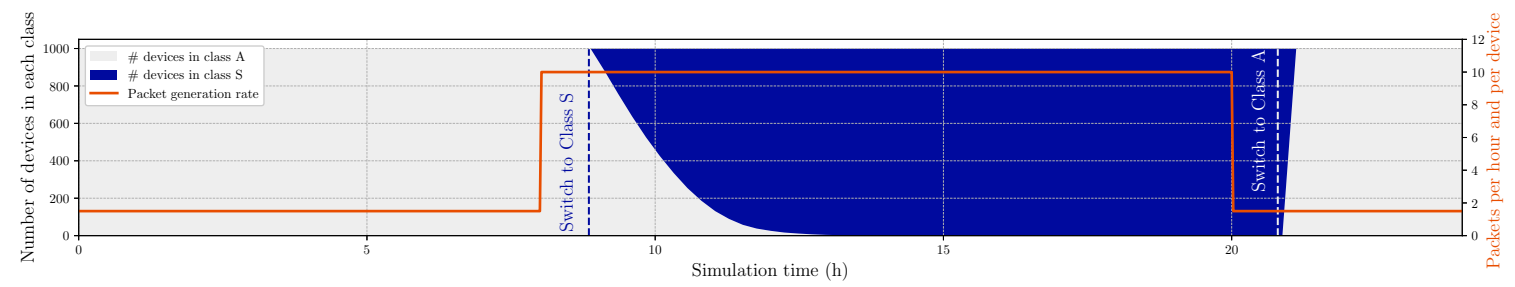

(a) Generation rate variation and evolution of the number of devices in each class, with TREMA enabled.

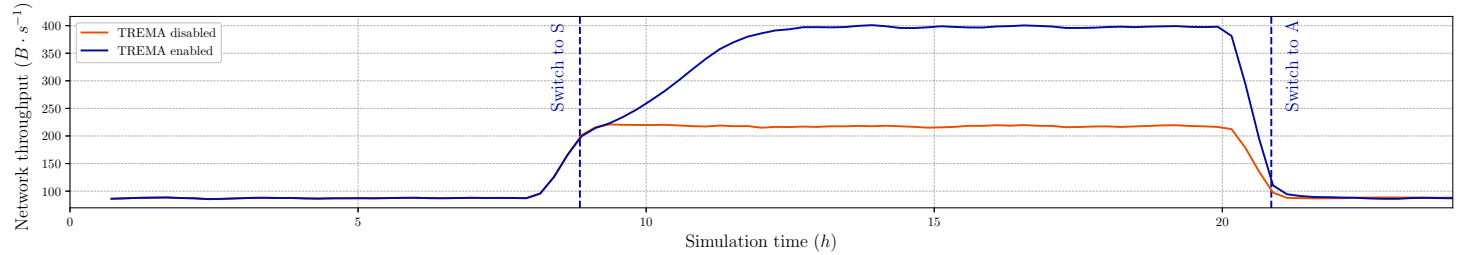

(b) Evolution of the $T$ over time, with and without TREMA.

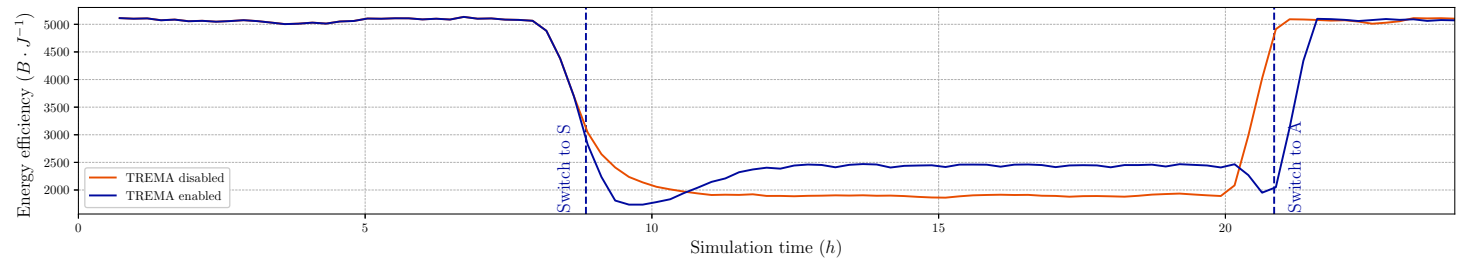

(c) Evolution of the $E$ over time, with and without TREMA.

Fig. 3: TREMA testing with one example scenario $\left(n_{\text {skip }}=0\right)$.

the fly for any network parameters. Research directions include probability models and machine-learning based approaches.

Further studies will also investigate the mitigation of network churn under unstable traffic conditions through hysteresis, assuring that a switching event is triggered only when observing a significant traffic load change for a sufficient amount of time, thus justifying the access scheme adaption. The impact of downlink data traffic on the performances will be assessed as well. Finally, a real-hardware implementation will also shed some light on the behavior of time-synchronized access schemes in real-world deployments, with the handling of device clock drifting and synchronization failures.

\section{REFERENCES}

[1] I. F. Akyildiz, W. Su, Y. Sankarasubramaniam, and E. Cayirci, "Wireless sensor networks: a survey," Computer Networks, vol. 38, no. 4, pp. 393-422, Mar. 2002. [Online]. Available: http://www.sciencedirect.com/science/article/pii/S1389128601003024

[2] LoRa Alliance, "LoRaWAN® Specification v1.1," 2017.

[3] N. Abramson, "THE ALOHA SYSTEM: another alternative for computer communications," in Proc. of AFIPS '70 (Fall), ser. AFIPS '70 (Fall). Houston, Texas: ACM, Nov. 1970, pp. 281-285.

[4] L. Chasserat, N. Accettura, and P. Berthou, "Short: Achieving energy efficiency in dense LoRaWANs through TDMA," in IEEE WoWMoM 2020, Cork, Ireland, Aug. 2020.

[5] P. I. Jalava, Q. Wang, K. Kuuspalo, J. Ruusunen, L. Hao, D. Fang, O. Väisänen, A. Ruuskanen, O. Sippula, M. S. Happo, O. Uski, S. Kasurinen, T. Torvela, H. Koponen, K. E. J. Lehtinen, M. Komppula, C. Gu, J. Jokiniemi, and M. R. Hirvonen, "Day and night variation in chemical composition and toxicological responses of size segregated urban air PM samples in a high air pollution situation," Atmospheric Environment, vol. 120, pp. 427-437, Nov. 2015. [Online]. Available: http://www.sciencedirect.com/science/article/pii/S1352231015303332
[6] C. Pham, "Robust CSMA for long-range LoRa transmissions with image sensing devices," in 2018 Wireless Days (WD), Apr. 2018, pp. 116-122.

[7] M. Rizzi, P. Ferrari, A. Flammini, E. Sisinni, and M. Gidlund, "Using LoRa for industrial wireless networks," in 2017 IEEE 13th WFCS, May 2017, pp. 1-4.

[8] T. Polonelli, D. Brunelli, A. Marzocchi, and L. Benini, "Slotted ALOHA on LoRaWAN-Design, Analysis, and Deployment," Sensors, vol. 19, no. 4 , p. 838 , Jan. 2019.

[9] J. Haxhibeqiri, I. Moerman, and J. Hoebeke, "Low Overhead Scheduling of LoRa Transmissions for Improved Scalability," IEEE Internet of Things Journal, vol. 6, no. 2, pp. 3097-3109, Apr. 2019.

[10] A.-I. Pop, U. Raza, P. Kulkarni, and M. Sooriyabandara, "Does Bidirectional Traffic Do More Harm Than Good in LoRaWAN Based LPWA Networks?" arXiv:1704.04174 [cs], Dec. 2017.

[11] R. Piyare, A. L. Murphy, M. Magno, and L. Benini, "On-Demand TDMA for Energy Efficient Data Collection with LoRa and Wake-up Receiver," in 2018 14th WiMob, Oct. 2018, pp. 1-4.

[12] L. Beltramelli, A. Mahmood, P. Osterberg, M. Gidlund, P. Ferrari, and E. Sisinni, "Energy efficiency of slotted lorawan communication with out-of-band synchronization," IEEE Transactions on Instrumentation and Measurement, p. 1-1, 2021.

[13] D. Zorbas, K. Abdelfadeel, P. Kotzanikolaou, and D. Pesch, "TS-LoRa: Time-slotted LoRaWAN for the Industrial Internet of Things," Computer Communications, vol. 153, pp. 1-10, Mar. 2020.

[14] K. Abdelfadeel, T. Farrell, D. McDonald, and D. Pesch, "How to make Firmware Updates over LoRaWAN Possible," arXiv:2002.08735 [cs], Feb. 2020, arXiv: 2002.08735.

[15] L. Leonardi, F. Battaglia, G. Patti, and L. L. Bello, "Industrial LoRa: A Novel Medium Access Strategy for LoRa in Industry 4.0 Applications," in IECON 2018 - 44th Annual Conference of the IEEE Industrial Electronics Society, Oct. 2018, pp. 4141-4146.

[16] Semtech Corporation, "AN1200.13 SX1272/3/6/7/8: LoRaModemDesigner'sGuide," 2013.

[17] E. Björnson and E. G. Larsson, "How energy-efficient can a wireless communication system become?" in 2018 52nd Asilomar Conference on Signals, Systems, and Computers, 2018, pp. 1252-1256. 